\title{
Éditorial : Les migrants dans l'espace transnational : permanence et changement
}

Editorial: Migrants in Transnational Space: Continuity and Change

Editorial: Los migrantes en el espacio transnacional: permanencia y cambio

Dietmar Loch et Jacques Barou

\section{(2) OpenEdition}

Journals

\section{Édition électronique}

URL : https://journals.openedition.org/remi/5714

DOI : $10.4000 /$ remi. 5714

ISSN : $1777-5418$

Éditeur

Université de Poitiers

Édition imprimée

Date de publication : 1 mars 2012

Pagination : 7-12

ISBN : 979-10-90426-03-0

ISSN : 0765-0752

\section{Référence électronique}

Dietmar Loch et Jacques Barou, «Éditorial : Les migrants dans l'espace transnational : permanence et changement », Revue européenne des migrations internationales [En ligne], vol. 28 - $n^{\circ} 1$ | 2012, mis en ligne le 25 juillet 2012, consulté le 14 avril 2022. URL : http://journals.openedition.org/remi/5714 ; DOl : https://doi.org/10.4000/remi.5714 


\section{Éditorial}

\section{Les migrants dans l'espace transnational : permanence et changement}

\section{Dietmar LOCH ${ }^{1}$ et Jacques BAROU ${ }^{2}$}

$D_{\mathrm{d}}$ epuis plusieurs années, le rapport entre migrations, transnationalisme et diaspora est exploré aussi bien dans le domaine des recherches empiriques que dans celui de la réflexion théorique, sans pour autant que se dégage un consensus sur la pertinence de ce lien ni sur le caractère véritablement novateur de sa prise en compte dans le champ des travaux sur les migrations internationales. De prime abord, on peut considérer qu'il s'agit peut-être là du nouvel habillage conceptuel d'une problématique aussi ancienne que les migrations internationales. Dès les années 1970, Abdelmalek Sayad (1999) insistait sur la nécessité de prendre en compte la question de l'émigré pour comprendre la condition de l'immigré. Il était pour lui impensable de faire abstraction du lien qui rattachait les immigrés au territoire dans lequel ils avaient été socialisés ou d'ignorer les trajectoires qu'ils avaient vécues avant leur installation dans le pays où ils résidaient. Il n'est pas nécessaire que ce lien soit réactivé par des séjours plus ou moins réguliers au pays pour qu'il puisse jouer un rôle dans la perception de la société de résidence, le positionnement de l'immigré par rapport à elle et les projets d'avenir qu'il y nourrit. Pour être de nature plus souvent idéelle que réelle, le lien entre les espaces de départ, de transit et d'arrivée n'en influence pas moins le rapport de «l'émigré-immigré » aux sociétés auxquelles il participe. Vivant dans plusieurs univers, il ne cesse de redéfinir son degré d'appartenance à ceux-ci. Il est souvent très présent intellectuellement et affectivement dans le monde dont il est le plus éloigné physiquement, se projetant vers son pays d'origine quand il est dans son pays d'accueil et cultivant la nostalgie de ce dernier quand il est de retour chez lui. Dans le lieu où il se trouve, il a toujours tendance à tisser des liens avec tout ce qui évoque le lieu où il ne se trouve pas. Il peut se définir essentiellement comme un être de paradoxe. Les systèmes de relations et les modes d'interaction qui constituent un des objets de recherche privilégié de l'analyse des sociétés contemporaines se révèlent particulièrement riches à observer dans les situations migratoires.

1 Maître de conférences en sociologie, Université de Grenoble II, UMR Pacte, CNRS ; dietmar. loch@upmf-grenoble.fr

2 Directeur de recherches CNRS, UMR Pacte, Institut d'Études Politiques de Grenoble, BP 48f, 38040 Grenoble cedex ; jacques.barou@iepg.fr 
La demande sociale a toutefois incité longtemps les chercheurs à se préoccuper essentiellement du devenir des populations immigrées au sein des sociétés où elles s'étaient installées, privilégiant ainsi les problématiques de l'adaptation, de l'insertion, de l'assimilation ou de l'intégration, que celles-ci soient en lien avec un processus d'évolution individuelle ou qu'elles s'articulent aux diverses philosophies du multiculturalisme. Cela a quelque peu contribué à laisser dans l'ombre les modes de participation des immigrés à la vie des sociétés dont ils n'étaient pas ou plus ressortissants alors qu'il est bien évident que dans de multiples cas, cette participation se maintenait, franchissant même la barrière des générations et reliant parfois les diverses communautés expatriées les unes aux autres à travers une commune référence à la terre ancestrale.

L'intérêt pour cette dimension transnationale de la migration s'est accéléré depuis les travaux pionniers de Nina Glick Schiller (1994), d'Alejandro Portes (1999) et de leurs collaborateurs, produisant même un « effet de mode » dans les sciences sociales. C'est pour dépasser cette dimension superficielle que nous avons souhaité publier un certain nombre d'articles apportant, dans la première partie de ce numéro, un regard critique sur la théorie, les concepts et les méthodologies utilisés dans ce champ de recherche encore récent. Dans ce contexte, il convient d'abord de s'interroger sur les raisons de l'avènement d'une telle problématique. On peut y voir, comme Chantal Bordes-Benayoun l'observe, une conséquence de l'épuisement des problématiques traditionnelles de la migration et de l'ethnicité, mais aussi le résultat des bouleversements qui ont affecté le monde avec la globalisation des échanges et, depuis la chute du mur de Berlin, avec les nouvelles dynamiques internationales. Ceci aurait incité les chercheurs à essayer de dépasser les frontières habituelles assignées aux objets sociologiques ou anthropologiques, qu'il s'agisse de groupes sociaux, de cultures ou d'espaces. Mais, comme c'est souvent le cas lorsqu'une nouvelle problématique apparaît dans le champ des sciences sociales, le manque d'outils existant pour l'aborder de manière pertinente entraîne d'inévitables risques de confusion et ce, d'autant plus que, parallèlement, le discours idéologique et politique s'empare inévitablement des notions mises sur le devant de la scène, leur donnant un sens indéfiniment extensible qui les rend inopérantes sur le plan scientifique. C'est ce qui arrive selon Chantal Bordes-Benayoun avec la notion de diaspora qui, après avoir désigné pendant longtemps des processus historiques de dispersion de populations bien identifiées, se décline désormais à l'infini, pour dénommer tout ce qui peut unir des gens dispersés autour d'une référence commune, qu'il s'agisse d'un pays d'origine, d'une identité régionale ou religieuse, d'un statut social particulier ou d'une combinaison de tout cela à la fois. Ce succès du concept, popularisé entre autres par les discussions et les diffusions via Internet, peut entraîner chez les chercheurs eux-mêmes une fascination les poussant à y recourir de façon pas toujours pertinente.

C'est pour prévenir un tel risque que Paolo Boccagni se livre ici à un examen approfondi des diverses notions que l'on voit graviter autour de la problématique du « transnational ». Constatant qu'en dépit de la place prise par la notion de transnationalisme dans les études sur les migrations, beaucoup de désaccords persistent à son sujet dans le monde de la recherche, il tente de lui donner une assise sociologique plus solide. Pour cela, il propose de définir de manière empiriquement pertinente cette notion, de la confronter avec les concepts utilisés dans le champ plus large des travaux sur les migrations internationales puis de se pencher plus avant sur la manière dont les pratiques trans- 
nationales s'inscrivent dans la trajectoire de vie des migrants. L'enjeu est de savoir si le transnationalisme est seulement une catégorie académique ou s'il peut faire sens pour les migrants eux-mêmes. Pour Paolo Boccagni, il convient tout autant de dépasser une problématique trop large du transnationalisme qui n'apporte rien de nouveau par rapport à ce qui est observé depuis longtemps à propos des migrations internationales comme d'éviter de s'enfermer dans une problématique trop étroite qui fait de l'approche transnationale un secteur trop délimité de la recherche sur les migrations pour influer sur sa compréhension globale. La question cruciale est de savoir comment s'articule le passage d'un mode de relation fondé sur la proximité physique à un mode de relation tout aussi intime et qui se développerait de façon durable en dehors de toute coprésence. C'est sur cette question que les sociologues des migrations auront, selon lui, à réfléchir dans l'avenir.

Si l'approche transnationale a conquis sa place au sein des recherches sur les migrations, elle a besoin d'un ensemble méthodologique dont Thomas Faist trace les principales caractéristiques. Considérant que cette méthode est moins déterminée par une discipline particulière des sciences sociales que par le champ de recherche que constitue le transnationalisme, il propose d'abord de définir plus rigoureusement trois notions associées à ce terme quelque peu attrape tout. Tout d'abord, la transnationalisation implique des relations transfrontalières régulières entre des individus ou des collectifs de personnes, aussi bien des migrants que des entreprises ou des communautés religieuses. Ensuite, les espaces sociaux transnationaux sont constitués par un enchaînement continu de liens et de pratiques transnationales mettant en action des réseaux internationaux de diverse nature. Enfin, la « transnationalité » lui apparaît comme un continuum de liens et de pratiques trans-étatiques de degré plus ou moins intense. Une méthode bien adaptée au champ de recherche transnationale doit se garder d'abord d'essentialiser des notions comme l'État, la nationalité ou l'ethnie et les chercheurs doivent s'interroger sur leur position propre qui ne peut être la même selon qu'ils sont eux-mêmes issus de pays d'émigration ou d'immigration.

Afin de tenir compte de la particularité du champ transnational, Thomas Faist prône une approche qualitative inspirée d'une ethnographie "multi-située », ou d'une ethnographie mobile, citant plusieurs exemples de recherches empiriques ayant pris pour objet une même population appréhendée dans différents lieux. Cela lui paraît toutefois insuffisant, car cette approche ne permet pas d'extrapoler les résultats obtenus. Il faudrait, pour cela, passer à une phase quantitative et surtout disposer d'éléments comparatifs, en particulier d'études concernant des populations similaires qui n'ont pas de pratiques transnationales, et, enfin, disposer si possible d'une profondeur historique pour analyser les liens transfrontières à diverses époques de leur mise en œuvre.

Les pratiques transnationales et les processus d'intégration, étudiés à travers des cas concrets, constituent une autre dimension de la problématique développée dans la deuxième partie de ce numéro. Les recherches présentées ici par Thomas Lacroix, Catherine Delcroix et Daniel Bertaux, ainsi qu'Eveline Reisenauer et Jürgen Gerdes, offrent de nombreux points communs. Les pratiques transnationales analysées dans ces trois articles dépassent largement le cadre des échanges traditionnels maintenus par les communautés immigrées avec leurs pays d'origine. Qu'il s'agisse des associations villageoises de Panjabis implantées en Grande-Bretagne ou des associations berbères d'Algérie ou du Maroc implantées en France, des associations de femmes marocaines 
constituées à Bruxelles ou de migrants turcs de diverses générations et de divers profils sociaux vivant en Allemagne, on observe un lien entre pratiques transnationales et intégration réussie dans les sociétés d'accueil. Cela va à l'encontre d'une opinion encore relativement répandue chez les décideurs en charge de l'immigration selon laquelle le processus d'intégration à la société de résidence serait exclusif du maintien de relations intenses avec le pays d'origine. Qu'il s'agisse de contribuer au développement des zones de départ, d'y mener des actions humanitaires ou d'y créer des entreprises filiales de celles créées dans le pays de résidence, il faut toujours bénéficier d'un capital social relativement important et d'une reconnaissance des institutions du pays d'origine avec lesquelles on est de toute façon obligé d'établir des liens de partenariat. Les recherches présentées ici montrent aussi que seules des personnes appartenant à une élite issue des migrations de travail peuvent prétendre jouer un rôle de leader dans ces activités transnationales. Elles ont pu accéder à cette position par la réussite économique réalisée dans le pays d'accueil comme certains entrepreneurs turcs ou d'origine turque en Allemagne, par l'accès à des responsabilités associatives importantes comme dans le cas des Marocains et des Algériens en France ou par des mandats politiques d'élus locaux dans le cas des femmes marocaines en Belgique. Ces personnes font preuve d'une activité économique et sociale et d'une conscience citoyenne qui transcende les frontières. Ce sens d'un intérêt et d'une responsabilité transnationale peut franchir la barrière des générations comme le prouve la trajectoire de certains citoyens allemands d'origine turque.

Toutefois, il convient de ne pas oublier quelques évidences générales qui permettent l'extension et l'enracinement dans le temps de telles pratiques. La relative proximité géographique et l'appui institutionnel apporté par les autorités du pays de résidence et parfois celles du pays d'origine ne sont pas sans incidence sur les pratiques transnationales. La densité des relations entretenue par les pays de l'Union européenne avec leur périphérie turque ou nord-africaine explique l'importance et la variété des échanges réalisés par les migrants en provenance de ces zones et certains de leurs descendants. En revanche, quand les distances sont plus grandes et les liens interétatiques moins denses, on observe que le transnationalisme se limite aux transferts de fonds et aux projets « classiques » de construction de maisons ou d'acquisition de biens dans le pays de départ et qu'ils déclinent ensuite avec les générations nées dans le pays d'accueil. C'est ce qu'observe Paolo Boccagni dans le cas des migrants équatoriens en Italie et Jacques Barou avec les migrants d'Afrique subsaharienne en France et au Royaume-Uni.

À un niveau plus large, les transferts de fonds restent de loin la principale manifestation de l'activité transnationale des migrants, atteignant 325 milliards de dollars en 2010. Dans la troisième partie du numéro, consacrée aux diverses formes du lien diasporique, Marie Coiffard analyse ces transferts de fonds à partir des outils de l'économie politique internationale. Devenus au cours des dernières années une des principales sources de devises des pays en développement, alors même que l'aide publique au développement est en forte baisse, les fonds transférés par les migrants éveillent de plus en plus l'intérêt des grandes institutions multilatérales et régionales. Toutefois, l'auteur identifie un certain nombre d'obstacles au passage à un véritable régime international de gestion de ces fonds. Le manque de régulation des transferts à un niveau global en est un des principaux. Il permettrait pourtant de réduire les coûts de ces transferts qui sont supportés essentiellement par les migrants. La seule initiative lancée l'a été en 2004 par le G8 sous le nom 
de « Global Remittance Initiative ». Cependant, l'envergure de cette initiative est limitée, car elle correspond plus à une normalisation des pratiques liées à une aversion commune des États impliqués pour l'absence de règles qu'à la résolution d'un problème d'action collective. De plus, ce régime international de gestion des transferts ne peut s'appuyer sur un équivalent international au niveau de la gestion des flux migratoires puisque celui-ci n'existe pas. Malgré ces effets limités, cette initiative a tout de même permis de repenser la problématique du développement en y intégrant l'apport des migrants.

Cet apport n'est pas systématique, comme l'observe Guillermo Uribe. Il distingue au sein de l'immigration latino-américaine en Espagne, d'une part, une migration de travail, provenant de pays généralement pauvres et qui va garder des liens forts avec sa zone de départ et y réaliser des transferts de fonds et des projets économiques et, d'autre part, une migration de caractère individuel qui n'exprime aucune préoccupation particulière vis-à-vis du pays d'origine et développe dans la société d'accueil des réseaux fondés sur une proximité sociale sans lien avec une appartenance communautaire. Ces divergences de trajectoire expliquent que, malgré la présence de ressortissants de presque tous les pays latino-américains en Espagne, on n'observe pas l'émergence d'une conscience de lien diasporique entre les migrants.

C'est ce que constate aussi Jacques Barou à propos des migrants sub-sahariens en Europe. Pour les premières générations, on observe le maintien d'un attachement fort au pays d'origine et la persistance d'une affirmation identitaire qui se réfère à l'appartenance nationale, voire à l'appartenance ethnique. Au fil du temps, celle-ci est amenée à composer avec une référence de plus en plus marquée au pays de résidence. Les générations nées dans les pays d'accueil se construisent une identité qui doit plus à la particularité du cadre national dans lequel ils vivent qu'à l'héritage africain plus ou moins bien transmis par les parents. De ce fait, si en France, on constate surtout une volonté d'intégration individuelle, tempérée par la sensibilité aux discriminations et par le désir de ne pas oublier d'où l'on vient, en Angleterre on observe plutôt un investissement dans des communautés religieuses, chrétienne ou musulmane, voire dans une communauté de « couleur », fondée davantage sur la distance cultivée vis-à-vis de la majorité blanche que sur le partage d'un héritage culturel commun aux populations noires.

Sur certains aspects, l'article rejoint l'idée développée par Chantal BordesBenayoun sur la nécessité de distinguer la diaspora noire en tant que projet politique porté par certaines élites afro-antillaise et la réalité sociale multiple et hétérogène vécue par les migrants et leurs descendants. La dimension diasporique s'affirme dans l'espace virtuel d'Internet où se multiplient les sites dédiés aux diasporas africaines ou à diverses visions de la diaspora noire, mais s'exprime peu dans la réalité des pratiques observées chez les migrants. Les constructions identitaires disposent d'une très grande marge de choix dans laquelle les influences du milieu de résidence, de la culture qui y est dominante et d'offres idéologiques et politiques qui s'y trouvent conservent une part prépondérante.

Ce numéro aura-t-il contribué à éclairer la question complexe du rapport entre migrations, transnationalisme et diaspora ? Nous espérons que la diversité des approches présentées ici aura au moins permis de poser un regard critique sur un phénomène appelé à se développer dans l'avenir et qui exigera de disposer des outils conceptuels et méthodologiques adéquats pour être abordé scientifiquement. 


\section{Références bibliographiques}

BASCH Linda, GLICK SCHILLER Nina and BLANC-SZANTON Cristina (1994) Nations Unbound: Transnational Projects, Postcolonial Predicaments and Deterritorialized Nationstates, Bâle, Gordon \& Breach Publishers, 335 p.

PORTES Alejandro, GUARNIZO Luis and LANDOLT Patricia (1999) The study of transnationalism: pitfalls and promise of an emergent research field, Ethnic and Racial Studies, 22 (2), pp. 217-37.

SAYAD Abdelmalek (1999) La double absence. Des illusions de l'émigré aux souffrances de l'immigré, Paris, Le Seuil, 448 p. 